\title{
Column generation for frequency assignment in slow frequency hopping networks
}

\author{
Arie Koster and Martin Tieves ${ }^{*}$
}

\begin{abstract}
This article deals with the frequency assignment problem (FAP) in slow frequency hopping (GSM) networks, a generalization of the classical FAP. Due to symmetry in the solutions, a natural integer linear programming formulation does not yield a good solution procedure. Instead, we decompose the co-channel and adjacent channel interference minimization and develop a two-stage algorithm. The co-channel optimization problem is solved with a column generation model, whereas the second stage is solved by a cutting plane approach. Computational experiments reveal, that although no optimal solutions can be guaranteed, the approach provides promising results, both regarding practical applicability and further research potential.
\end{abstract}

Keywords: Slow frequency hopping, Interference minimization, Column generation

\section{Introduction}

Mobile communication, as a key aspect of modern society, looks upon a history of success and growth. Consequentially, a wide range of organizational questions, connected to a network's structure and usage, have been in the focus of research ever since. This article concentrates on frequency planning. Mobile communication physically takes place in the radio spectrum. This spectrum is a limited resource, it can only support a finite number of frequencies. Consequently, a sensible assignment of these frequencies to signal emitters is necessary. On the background of GSM technology or modified for more modern standards like WLAN, UMTS, and LTE, the settings of the classical frequency assignment problem (FAP) have commonly been accepted as an insightful point of research for questions related to FAPs. FAP focuses on the optimization of mobile communication quality by means of minimizing mutual (co- and adjacent channel) interference between signal transceivers (TRX).

Clearly, the FAP is a highly abstracted model of reality. In this article, the difficulties of obtaining these (interference) values and other influencing factors of communication quality among power control techniques, adaptive modulation, or channel utilization are not treated in

*Correspondence: Tieves@math2.rwth-aachen.de Lehrstuhl 2 für Mathematik, RWTH, Aachen, Germany detail. However, especially the topics adaptive modulation and power-control play important roles in resource management and are closely related to questions of transmission quality since both have a direct impact on interference measures. We refer to [1-3] for in-depth information on adaptive modulation and to $[4,5]$ for information on power-control techniques. In this article, we focus on the abstract mathematical optimization problem and assume these influences sufficiently clarified beforehand.

While the FAP considers a static assignment of frequencies to signal emitters (one channel per TRX), the concept of slow frequency hopping (SFH) is going beyond this one-to-one mapping and assigns multiple frequencies to each TRX. In SFH, a TRX does not transmit permanently on one frequency, but changes its transmitting frequency within a specific frequency pool at random periods. Hereby, the main goal of FAP persists: minimizing the total expected (mutual) interference of these assignments. The focal point of SFH, randomization, incorporates statistical effects, leading to interference and frequency diversity. Basically, this means that frequency-related characteristics are averaged. For example, signal-spreading is highly frequency related, such that the extreme peeks in signal distribution are cut off by SFH on average. Signals at a specific location are no longer either very bad (unusable) or very good (inefficient) the whole time, though bad conditions on isolated

\section{Springer}

(c) 2012 Koster and Tieves; licensee Springer. This is an Open Access article distributed under the terms of the Creative Commons Attribution License (http://creativecommons.org/licenses/by/2.0), which permits unrestricted use, distribution, and reproduction in any medium, provided the original work is properly cited. 
time frames are still very possible since they can be easily corrected via error recognition codes. Here we refer to [6-8] for more information. Thus, randomization effectively lowers the network's signal-to-noise ratio, resulting in an increased capacity or improved transmission quality compared to non-hopping networks. In the following, this problem is denoted with SFH-FAP.

In this study, the problem of finding frequency assignments for SFH networks is formulated by means of mixed integer programming. Hereby, SFH-FAP is interpreted as an advancement of FAP (see [9] for a survey on FAP). Since a column generation approach is invoked here, the work of Jaumard (and others) is to mention, for example [10-12]. There, a similar problem is analyzed, enriched with the aspects of antenna spacing but reduced to interpreting interference values not as floating numbers but only as integer levels (interference possible from 0 to 10). Performing column generation as well, their approach is closely related to the graph coloring problem in Mehrotra and Trick [13].

Concerning SFH-FAP, frequency planning has been done on a heuristic basis, mostly. Here one could mention the papers [14-16]. There, frequency assignments are created by the means of frequency lists for each transceiver, which are created and improved on a heuristic basis (e.g., by simulated annealing). While these methods are usually fast, they cannot claim to provide optimal frequency assignments. In contrast to these approaches, this article tries to invoke exact methods for obtaining optimal frequency assignments. Therefore, advanced optimization concepts like column generation and cutting plane algorithms are used. Though frequency assignments can be provided, no guarantees of optimality can be given. Nevertheless, this article analyzes the usage of the chosen means and points out future research potential. On the whole, this study is based on the master thesis of Tieves [17].

In the following, the problem description is formalized and a mixed integer formulation is given. Next, the problem is decomposed into two sub-problems, treated consecutively. The resulting optimization procedure is evaluated for modified test examples from the FAP website [18]. At the end of this article, some conclusions of this approach as well as future research proposals are given.

\section{Problem description}

First, the input of the classical FAP is briefly summarized and the SFH-FAP input is evolved from this. A mathematical problem description is presented, leading to a mixed integer formulation of this problem at the end of the section.

\section{Frequency assignments in GSM networks}

A detailed description of the FAP problem in GSM networks can be found in [19]. There, the FAP is deeply explained and information are given, how the model, i.e., the interference values can be derived from reality. Here we focus on the abstracted mathematical optimization problem and invoke no deepening research on these matters.

From a mathematical perspective, an instance of the FAP can be interpreted as an undirected graph $G=(\mathcal{T}, E)$ and a set $F$ of available frequencies. We assume that the frequencies are equally spaced in the radio spectrum. The vertices $v \in \mathcal{T}$ represent the signal emitters (TRXs). For every edge $e=\left\{v_{1}, v_{2}\right\} \in E$ with $v_{1}, v_{2} \in \mathcal{T}, \operatorname{co}\left(v_{1}, v_{2}\right) \geq 0$ and $a d\left(v_{1}, v_{2}\right) \geq 0$ define the amount of co- and adjacent channel interference, which is induced if both TRXs transmit on the same, respectively, neighboring frequencies. Further a separation distance $d_{v_{1}, v_{2}} \in \mathbb{N}$ might be defined for edges $\left\{v_{1}, v_{2}\right\} \in E$, specifying the minimum distance between the transmissions of $v_{1}$ and $v_{2}$ in the frequency spectrum. For every vertex $v \in \mathcal{T}$, a set of (locally) blocked frequencies $B_{v} \subset F$ might be defined, containing the frequencies which cannot be assigned to this TRX. The aim is to find a frequency assignment, i.e., a mapping of one frequency $f \in F$ to every TRX $v \in \mathcal{T}$, such that the separation and blocking constraints are met and the total induced co- and adjacent channel interference is minimal. In this context, the interference values can be interpreted as costs or penalties as well.

\section{SFH}

For a FAP-SFH instance, as a generalization of the FAP problem, we assume that two additional inputs are complementing the FAP input. First, a partition of the TRXs is provided, i.e., a collection $\mathcal{S}=\left\{I_{1}, \ldots, I_{p}\right\}$ with $I_{i} \subseteq \mathcal{S}$, $\bigcup_{i=1}^{p} I_{i}=\mathcal{T}$ and $I_{i} \cap I_{j}=\varnothing$ for all $i \neq j$. For each $i$ the set of TRXs $I_{i}$ is called super transceiver (STRX) in the following. Second, for every $I \in \mathcal{S}$ a value $k_{I} \in \mathbb{N}$ denoting the required amount of frequencies of this STRX is given. As an interpretation: Each TRX $v$ is in one STRX $I$ and at every time frame, $v$ chooses a frequency $f$ to transmit on, out of the $k_{I}$ available ones, at random uniformly and independently distributed. Hereby the STRX structure shows, which TRXs may potentially choose the same frequencies.

Now an assignment should be provided, which assigns $k_{I}$ frequencies to every STRX $I$ such that the quality is as good as possible, i.e., the total interference is as low as possible. However, the amount of interference in a FAP instance on a link between two TRXs can be measured any time, but the amount of interference between two STRXs $I$ and $J$ is not a fixed value any longer, due to the randomization concept of SFH. Judging an assignment's quality, various measures like expected-interference or worst-case interference are possible. Because of the frequency at which a TRX can change its frequency $(7.5 / 13 \mathrm{~ms}$, see [19]), we assume that the expected interference is a reasonable choice, since the user can only experience an 
averaged quality - the single time-frame cannot be distinguished by the human ear. Because of the randomization, in every single time-frame the frequency assignment produces a higher interference level compared to a FAP assignment, however the frequency hopping gain surpasses the higher average interference. Nevertheless, we assume the expected interference as a suitable measure for obtaining frequency assignments. For each pair of STRXs $I$ and $J$, these expected co- and adjacent channel interferences $c o(I, J)$ and $a d(I, J)$ can be determined as follows.

Given a frequency $f$ assigned to both STRX I and STRX $J$, the probability that two TRXs $v \in I$ and $w \in J$ choose this frequency simultaneously is $\frac{1}{k_{l} \cdot k_{J}}$, inducing a co-channel interference $c o(v, w)$. Hence, the expected cochannel interference is $c o(I, J) \cdot y_{I, J}$ where $y_{I, J}$ denotes the number of equal channels assigned to $I$ and $J$ and

$$
\operatorname{co}(I, J):=\sum_{v \in I} \sum_{w \in J} \frac{1}{k_{I} \cdot k_{J}} \cdot \operatorname{co}(v, w) .
$$

Similarly, the expected adjacent channel interference is $\operatorname{ad}(I, J) \cdot n_{I, J}$ where $n_{I, J}$ is the number of adjacent channels assigned to $I$ and $J$ and

$$
\operatorname{ad}(I, J):=\sum_{v \in I} \sum_{w \in J} \frac{1}{k_{I} \cdot k_{J}} \cdot \operatorname{ad}(v, w) .
$$

Summing up the differences between FAP and SFH-FAP, the interference on TRX level is aggregated to the STRX level such that the induced interference between two STRXs is the total (expected) interference of the inbound TRXs. Nevertheless, an SFH-FAP instance is similar to an FAP instance, with the difference, that for the nodes corresponding to an STRX more than one frequency per node is needed and the edge-weights are adapted in the above way (1), (2). The downside of this aggregation lies within the separation and blocking constraints. When aggregating the TRXs to the STRX level, separations may be specified between two STRXs (denoted with $d_{I, J} \in \mathbb{N}$ ) or between each TRX within a STRX (denoted by $d_{I} \in \mathbb{N}$ ). However, since the frequencies for each TRX in the STRX are drawn at random, no separation can be guaranteed on the inner TRX level any longer. Furthermore, separations on the STRX level hold for all TRXs within these STRXs such that not all constraints can be aggregated and those which are lifted to the STRX level may be more striking than before. Additionally, the same applies for the blocking constraints.

Note that these disadvantage arises from considering arbitrary STRX structures. Establishing a frequency hopping structure in a network, it is always possible to regard each single TRX as STRX. Then, all separations/blocking constraints can be transported into that framework. However, the mathematical consequence is a greatly enlarged problem size. In practical aspects, some trade-off between problem-size and correctness is reasonable. Incorporating SFH at the expense of some inaccuracy within the constraints may still improve the overall network quality.

As an example of this transformation, one may consider Figure 1. Given the set of TRXs as $\mathcal{T}:=\{1, \ldots, 7\}$, the set of all STRXs may be formed of three STRXs $\mathcal{S}:=$ $\left\{I_{1}, I_{2}, I_{3}\right\}$. In this example, $I_{1}$ consists of the TRXs one to three, $I_{2}$ contains four and five and STRX $I_{3}$ consists of the remaining TRXs. The relations (co-, adjacent channel interference, separations, and blockings) on TRX level, here shown as dotted, are aggregated to STRX level (red lines) via the procedure mentioned above. The randomization of SFH is incorporated in the STRX relations, the interference values are expected values and no fixed, measurable information any longer. For clearness sake, the interference relations "within" a single STRX have been omitted. Given a STRX $I, c o(I, I)$ is a constant for every frequency assigned, determined by the STRX structure, i.e., the inbound TRXs. For the expected co-channel interference, this constant is multiplied with the amount of frequencies to be assigned, an other constant $\left(y_{I, I}=k_{I}\right)$. As a result the co-channel interference value within a STRX is already determined by the STRX structure and independent from the specific frequency assignment. However, inner STRX adjacent channel interference is not a constant and can be determined as described in (2).

In summary, SFH-FAP aims to find an optimal frequency assignment, i.e., assign at least $k_{I}$ frequencies $f \in$ $F$ to every STRX $I$ such that the expected induced interference is minimal, all separation/blocking constraints are met and the totally induced expected co- and adjacent channel interference is minimal. This can be formalized as follows.

The input for the SFH-FAP problem is a nine-tuple $M:=\left(\mathcal{S}, E, F,\left\{B_{I}\right\}_{I \in \mathcal{S}}, k_{I}, d_{I}, d_{I, J}, c o, a d\right)$. A feasible solution, i.e., a feasible assignment, is a function

$$
Y: \mathcal{S} \rightarrow 2^{F}
$$

mapping each vertex to a set of frequencies such that

$$
\begin{array}{lll}
|Y(I)| & =k_{I} & \forall I \in \mathcal{S}, \\
Y(I) & \subseteq F \backslash B_{I} & \forall I \in \mathcal{S}, \\
\left|f_{1}-f_{2}\right| & \geq d_{I} & \forall I \in \mathcal{S}, f_{1}, f_{2} \in Y(I) \text { with } f_{1} \neq f_{2}, \\
\left|f_{1}-f_{2}\right| & \geq d_{I, J} & \forall\{I, J\} \in \mathcal{S} \times \mathcal{S}, f_{1} \in Y(I), f_{2} \in Y(J) .
\end{array}
$$

Given the above tuple and denoting the set of neighboring STRXs for two STRXs $I$ and $J$ by

$N_{I, J}(Y):=\left\{\left(f_{1}, f_{2}\right) \in F:\left|f_{1}-f_{2}\right|=1, f_{1} \in Y(I), f_{2} \in Y(J)\right\}$, 


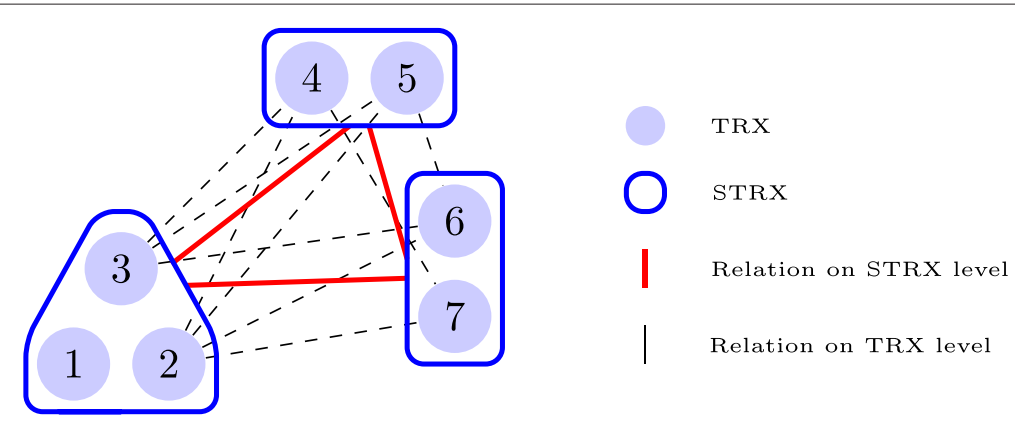

Figure 1 TRX to STRX graph. Shows the transformation from TRX to STRX level.

the FAP for SFH networks (FAP-SFH) is

$$
\begin{array}{r}
\min \left\{\sum_{I, J \in \mathcal{S}}|Y(I) \cap Y(J)| \cdot \operatorname{co}(I, J)+\sum_{I, J \in \mathcal{S}}\left|N_{I, J}(Y)\right|\right. \\
\cdot a d(I, J): Y \text { satisfies }(3 a)-(3 d)\} .
\end{array}
$$

\section{A mixed integer formulation}

Given the described input, the FAP-SFH problem can be formulated as a mixed integer linear program (MILP). The following variables are used:

$$
x_{I, f}=\left\{\begin{array}{l}
1, f \text { used at } I \\
0, \text { else }
\end{array} \quad \forall I \in \mathcal{S}, f \in F .\right.
$$$$
z_{I, J f f}=\left\{\begin{array}{l}
1, f \text { used at } I \text { and } J \\
0, \text { else }
\end{array}\right.
$$$$
\forall I, J \in \mathcal{S}, f \in F \text {. }
$$

$b_{I, J f}=\left\{\begin{array}{l}2, f \text { used at } I, \text { both } f+1 \text { and } f-1 \text { used at } J \\ 1, f \text { used at } I, \text { either } f+1 \text { or } f-1 \text { used at } J \\ 0, \text { else }\end{array}\right.$

$$
\forall I, J \in \mathcal{S}, f \in F \text {. }
$$

$y_{I, J} \hat{=}$ number of equal channels at $I$ and $J$.

$$
=\mid\{f \in Y(I) \mid f \in Y(J)\}
$$

$n_{I, J} \hat{=}$ number of adjacent channels at $I$ and $J$.

$$
=\mid\{f \in Y(I) \mid f+1 \vee f-1 \in Y(J)\}
$$

Note that this directed relations could be transformed into undirected relations as well. However, this leads to the following MILP:

$$
\begin{array}{ll}
\min \sum_{I \in \mathcal{S}} \sum_{J \in \mathcal{S}} c o(I, J) \cdot y_{I, J}+a d(I, J) \cdot n_{I, J} \\
\text { s.t. } \sum_{f \in F} x_{I, f}=k_{I} & \forall I \in \mathcal{S} \\
z_{I, J, f} \geq x_{I, f}+x_{J, f}-1 & \forall I, J \in \mathcal{S}, f \in F \\
\sum_{f \in F} z_{I, J f f}=y_{I, J} & \forall I, J \in \mathcal{S}
\end{array}
$$

$b_{I, J, f} \geq 2 \cdot x_{I, f}+x_{J, f-1}+x_{J, f+1}-2 \quad \forall I, J \in \mathcal{S}, f \in F$

$$
\sum_{f \in F} b_{I, J f f}=n_{I, J} \quad \forall I, J \in \mathcal{S}
$$

$$
x_{I, f_{1}}+x_{I, f_{2}} \leq 1 \quad \forall I \in \mathcal{S}, f_{1}, f_{2} \in F,\left|f_{1}-f_{2}\right| \leq d_{I}
$$

$$
x_{I, f_{1}}+x_{I, f_{2}} \leq 1 \quad \forall I, J \in \mathcal{S}, f_{1}, f_{2} \in F,\left|f_{1}-f_{2}\right| \leq d_{I, J}
$$

$$
x_{I, f}=0 \quad \forall I \in \mathcal{S}, f \in B_{I} .
$$

The objective function minimizes the total (expected) interference. Constraints (5a) ensure that every STRX gets enough frequencies. In (5b) and (5d), common- and adjacent channels are recognized. These channels are summed up in (5c) and (5e), respectively, for the number of equalor adjacent channels. Further, constraints (5f) and (5g) model separation requirements. Hereby constraints (5f) represent the separation requirements between the frequencies of one STRX (inner STRX separation) and constraints $(5 \mathrm{~g})$ describe the necessary separations between the frequencies of two different STRXs (outer STRX separation). Finally, constraints (5h) represent the blocked frequencies per STRX. Henceforth, this model is referred to as [FAPSFH]. 
While this formulation is straightforward, it bears some major drawbacks preventing computability for practical scenarios. The model consists of $O\left(|\mathcal{S}|^{2} \cdot|F|\right)$ variables and constraints of the same order. Bearing in mind that practical instances may have hundreds of STRXs and frequencies, memory restrictions are challenging. Further, a formulation with penalty variables, for example recognition of interferences via constraints of the type: $A \wedge$ $B \Leftrightarrow C$ like in (5b), (5d), is impracticable. Since the linear relaxation of such a formulation has a value of zero (due to the fact that the penalty variables are avoidable by fractional values on the decision variables), the solution process is effectively rendered to an enumeration of the integer solutions, which is inappropriate for realistically sized instances.

\section{Decomposition into two stages}

Since the above presented formulation is not applicable in practice, a reformulation by a Dantzig-Wolfe reformulation is a promising alternative. The resulting formulation has an exponential number of variables, implying that dynamic column generation has to be used. In the last decade or so, column generation methods have become very powerful to solve large-scale MILPs. In the case of FAP-SFH, column generation can be applied most effectively if the problem is first decomposed into two subproblems. For this purpose, the separation and blocking constraints are emitted and the interference calculations are ordered. First a minimum co-channel interference assignment is created, which is then adapted to minimize adjacent channel interference without increasing the co-channel interference. The results of this two stage approach are not equivalent to those of the [FAPSFH] model, but computability is enforced as a trade off.

In this approach, the first problem determines which STRXs share the same frequencies without specifying their actual frequencies, only with respect to co-channel interference minimization. Then, in the second stage, this assignment is re-optimized considering adjacent channel interference. This is possible because the co-channel interference is independent of the specific frequency, whereas the adjacent channel interference is derived only from the exact frequency allocation. If it is known which STRXs share how many frequencies, the co-channel interference can already be calculated, but for adjacent channel interference, the specific allocated frequencies have to be known.

\section{Co-channel interference minimization}

A problem description of the first stage problem can be obtained by restricting the SFH-FAP problem to cochannel interference (and omitting adjacent channel interference and separation/blocking constraints). Formally, the (restricted) input can be described as a six-tuple $Q:=$
$\left(\mathcal{S}, E, F, k_{I}, c o\right)$, with the same notations as above. Similar, the first stage problem is defined by

$$
\min :\left\{\sum_{I, J \in \mathcal{S}}|Y(I) \cap Y(J)| \cdot \operatorname{co}(I, J) \quad: \quad Y(I)=k_{I}\right\} .
$$

Since the total interference is only dependent on the size of the intersection of the assigned frequencies of each two STRX, it is sufficient to determine which STRX share how many frequencies. With this knowledge, one can state, which sets $T \subseteq \mathcal{S}$ of the STRXs share how many frequencies and derive the total co-channel interference of the assignment from this information. For example, information of the type "a set of STRXs $T:=\left\{I_{k}, I_{l}, I_{m}\right\}$ shares two frequencies" means that two of the available frequencies are assigned to each of the inbound STRXs of $T$ but to no other STRX. If this information is available for the complete number of frequencies, the co-channel interference can be determined, i.e., the exact common frequencies are not of interest (we refer to [17] for more details). Consequentially, the problem basically boils down to a vertex multi-coloring problem with a limited number of colors, i.e., the frequencies [20]. This problem can be described via the following MILP, where $x_{T} \in \mathbb{Z}_{+}$denotes how many frequencies the STRXs in $T$, and only those in $T$, have in common. Note that the corresponding dual variables $(\pi)$ are shown as well, as they are needed in the following.

$$
\begin{aligned}
\min & \sum_{T \subseteq \mathcal{S}} \operatorname{co}(T) \cdot x_{T} \\
\text { s.t. } & \sum_{T \subseteq \mathcal{S}} x_{T}=k_{I} \quad \forall I \in \mathcal{S}\left[\pi_{I}\right] \\
& \\
& \sum_{T \subseteq T} x_{T}=|F|\left[\pi_{0}\right]
\end{aligned}
$$

The amount of induced interference of each set $T$ is denoted by $\operatorname{co}(T)$, which is calculated by means of the STRX interference values as

$$
\begin{aligned}
\operatorname{co}(T):= & \sum_{I \in T} \sum_{J \in T} \operatorname{co}(I, J)=\sum_{I \in T} \sum_{J \in T} \sum_{\nu \in I} \sum_{w \in J}\left(\frac{1}{k_{I}} \cdot \frac{1}{k_{J}}\right) \\
& \times \operatorname{co}(\nu, w) .
\end{aligned}
$$

For the sake of completeness it is mentioned, that the constraints (6b) and (6c) can be formulated as inequalities, with $\geq$ and $\leq$, respectively, as well. Assume an optimal solution given, whereas one constraint $I$ of $(6 \mathrm{~b})$ is not fulfilled with equality. So STRX $I$ can be omitted in at least one set $T$, for which holds $I \in T, x_{T}>0$. By co-channel interference definition, it holds that $\operatorname{co}\left(T_{1}\right) \leq \operatorname{co}\left(T_{2}\right)$ if $T_{1} \subseteq T_{2}$, the induced new solution fulfills this constraint with equality and is at least as good as the first solution. Similar applies to constraint (6c). Assume one frequency 
was not used, it is always possible to split a set $S$ into two sets $S_{1}$ and $S_{2}$ with $S_{1} \cup S_{2}=S, S_{1} \cap S_{2}=\emptyset$ and adapt the solution via $x_{S}=0$ and $x_{S_{1}}=x_{S_{2}}=1$. Again by definition, $\operatorname{co}(S) \geq \operatorname{co}\left(S_{1}\right)+\operatorname{co}\left(S_{2}\right)$, such that the new solution, which fulfills (6c) with equality, is optimal as well. As a result, even when formulating via inequalities, there is always an optimal solution, which would satisfy the equalities as well. Since the formulation via equalities is more intuitive, we stick to this formulation in the following.

Note that this model, denoted with [FAPH1], has significantly less constraints than the [FAPSFH] model but exponentially many variables. Before going into detail, the example presented in Section "SFH" is extended for clearness sake. [FAPH1] contains variables for every subset of $\mathcal{S}=\left\{I_{1}, I_{2}, I_{3}\right\}$. Assume that potential separation and blocking constraints have been omitted like described above and that

$$
\begin{array}{lll}
k_{I}=2 & \forall I \in \mathcal{S}, & \\
F=\{1,2,3,4\}, & \operatorname{co}\left(\left\{I_{i}\right\}\right)=0 & \forall i \in\{1,2,3\}, \\
& \operatorname{co}\left(\left\{I_{i}, I_{j}\right\}\right)=1 \quad \forall i, j \in\{1,2,3\}, \\
& \operatorname{co}\left(\left\{I_{1}, I_{2}, I_{3}\right\}\right)=3 . &
\end{array}
$$

Obviously, every solution will induce an interference value strictly above zero, since at least two STRXs will interfere each other. An optimal solution of the LP relaxation, which is already integer, is given by

$$
\begin{aligned}
& x_{\left\{I_{1}, I_{2}\right\}}=x_{\left\{I_{3}\right\}}=2 \\
& x_{\left\{I_{1}\right\}}=x_{\left\{I_{2}\right\}}=x_{\left\{I_{2}, I_{3}\right\}}=x_{\left\{I_{1}, I_{3}\right\}}=x_{\left\{I_{1}, I_{2}, I_{3}\right\}}=0 .
\end{aligned}
$$

Therefore, STRX $I_{3}$ is assigned two frequencies and STRXs $I_{2}$ and $I_{3}$ have two other (but each the same) frequencies assigned. However, the specific frequencies have not been determined, yet.

On a side note, this example visualizes the strength of [FAPH1] over the [FAPSFH] formulation. In the linear relaxation of [FAPSFH], the solution $x_{I, f}=0.5$ for all TRX $I$ and all frequencies $f$ is optimal, and extended to the other variables, has an objective value of zero, opposed to the LP-solution of [FAPH1] presented above. Consequently, the [FAPH1] formulation is preferable, since the solution of its linear relaxation is in general closer to the integer solution value.

Nevertheless, the [FAPH1] formulation has the disadvantage, concerning computational accessibility, of exponentially many variables. This is a classical setting for column generation to solve the linear relaxation (LP). In column generation only a subset of all possible variables is initially used and loaded into memory. The linear program is solved on that subset and it is determined, whether a variable (outside of that subset) with negative reduced costs exists. If such a variable can be found, it is added to the already known variables and the process starts again. If no improving variable can be found, the LP solution must be optimal. The critical point in this approach is the problem of finding variables with negative reduced costs, without generating the corresponding variable (column) beforehand. This problem is called the pricing problem. On a theoretical view, if the pricing problem can be solved in polynomial time, the LP relaxation of (6a)-(6c) can be solved in polynomial time [21]. However, the stable set problem, which is NP-complete [22], can be reduced to the pricing problem of [FAPH1]. Nevertheless, the advantage of this procedure is that it is possible to work on a subset of all variables (from a computational point of view), only, avoiding memory restrictions because of theoretically exponentially many variables. For a survey on column generation, see [23] or for the related vertex coloring example [13].

For such a column generation approach, a starting solution with a set $T$ containing all variables is feasible, i.e., all STRXs are mapped to the same set of frequencies; hence, the solution will have very high costs. Then, additional variables/sets are added as long as variables with negative reduced costs can be found. Given a feasible (LP) solution and the corresponding dual values $\pi$, the proof that such variables exist can be derived from the pricing problem [FAPHPR]:

$$
\begin{array}{lr}
\min \sum_{I \in \mathcal{S}} \sum_{J \in \mathcal{S}} c o(I, J) \cdot z_{I, J}-\sum_{I \in \mathcal{S}} \pi_{I} \cdot x_{I}-\pi_{0} \\
\text { s.t. } z_{I, J} \geq x_{I}+x_{J}-1 & \forall I, J \in \mathcal{S}, \\
z_{I, J} \in\{0,1\} & \forall I, J \in \mathcal{S}, \\
x_{I} \in\{0,1\} & \forall I \in \mathcal{S} .
\end{array}
$$

This MILP determines a set $T$ with minimal reduced cost. The constraints model a variable of the original LP, i.e., a set of STRXs and the objective function indicates the reduced costs, namely this variable's original objective coefficient minus the product of the dual values and the corresponding matrix column. Hereby, the variables $x_{I}$ determine, similar to a characteristic vector, whether STRX $I$ is contained in $T$ and the $z_{I, J}$ calculate the induced interference for the corresponding objective coefficient.

A variable with negative-reduced costs exists if and only if the MILP has a solution with an objective value strictly lower than zero. In this case the variable/set is given by the characteristic vector $x$, namely $T:=\left\{I \in \mathcal{S}: x_{I}=1\right\}$. Note that any solution with negative reduced costs is sufficient for creating a new variable, which might improve the current solution. Since the reduced costs measure the relative improvement from one basis solution to another, but not the quality gain towards the optimal solution, no predictions concerning the long-run performance of the new variables can be made on basis of the reduced costs. 
Hence, a heuristic solution of [FAPHPR] to find a negative reduced cost variable is usually sufficient. Only to guarantee LP optimality in the end, [FAPHPR] has to be solved to optimality.

By the above described procedure, the LP relaxation can be solved. For obtaining optimal integer solutions pricing has to be combined with a branch and bound algorithm. Hereby it might be needed to add new variables in each node, again. Combined, this approach is called branch $\mathcal{E}$ price.

\section{Adjacent channel interference minimization}

In the following, we assume a solution $\bar{T}:=\{T \subseteq \mathcal{S}:$ $\left.x_{T}>0\right\}$ for the first stage problem given. In this solution, the induced co-channel interference is already determined together with the sets of STRXs sharing the same frequencies. For obtaining a final assignment, specific frequencies have to be assigned to these sets such that the induced adjacent channel interference is minimal. Formally, the input can be given as a four-tuple $R:=\left(\bar{T}, F, x_{T}, a d\right)$. Here $F$ and $\bar{T}$ are used as described above. Furthermore, for every set $T \in \bar{T}$ the value $x_{T}$ from the first stage problem denotes the amount of frequencies, which should be assigned to $T$. The adjacent channel interference values between two sets $S, T \in \bar{T}$ (note: not necessarily $S \neq T$ ) can be aggregated similar as the co-channel interferences via

$$
\begin{aligned}
\operatorname{ad}(S, T):= & \sum_{I \in S} \sum_{J \in T} a d(I, J)=\sum_{I \in S} \sum_{J \in T} \sum_{v \in I} \sum_{w \in J} \frac{1}{k_{I} \cdot k_{J}} \\
& \times a d(v, w) \quad \forall S, T \in \bar{S} .
\end{aligned}
$$

A feasible solution/assignment to this input is a function

$$
\bar{Y}: \bar{T} \rightarrow 2^{F},
$$

mapping each set uniquely to one or more frequencies. Then, the second stage problem is defined by

$$
\min \left\{\begin{array}{c}
\sum_{S \in \bar{T}} \sum_{T \in \bar{T}}\left|N_{S, T}(\bar{Y})\right| \cdot \operatorname{ad}(S, T):|\bar{Y}(T)|=x_{T} \\
\forall T \in \bar{T}, \bar{Y}(T) \cap \bar{Y}(S)=\emptyset \quad \forall S, T \in \bar{T}
\end{array}\right\},
$$

whereas $N_{S, T}(\bar{Y})$ denotes the set of adjacent channels from $S$ in $T$ in the assignment $\bar{Y}$ via

$$
N_{S, T}(\bar{Y}):=\left\{\left(f_{1}, f_{2}\right) \in F:\left|f_{1}-f_{2}\right|=1, f_{1} \in \bar{Y}(S), f_{2} \in \bar{Y}(T)\right\},
$$

the above problem can be formulated as traveling salesman problem (TSP), as follows. To explain, first let us assume that every set $T$ of STRXs has only one frequency in common $\left(x_{T}\right.$ binary, see below for an analysis of the general case). Each set $T$ is now interpreted as a vertex in a new graph $(\bar{T}, F)$. Each of these vertices is connected to all other vertices with the induced adjacent channel interference as the weight. Furthermore, a dummy vertex with distance zero to all other vertices is added. Recall that adjacent channel interference is determined by the information which STRXs transmit on neighboring frequencies. So, each frequency can only interfere with two other frequencies and the interference is only dependent on the STRXs mapped to these frequencies. For example, frequencies 4 and 5 induce the same adjacent channel interference as frequencies 8 and 9 between the same STRXs. As a consequence, finding an assignment with minimal adjacent channel interference is equivalent to finding a shortest tour visiting all vertices in the constructed graph. The sequence of vertices visited corresponds to the index of the assigned frequency. Starting from the dummy vertex, all STRXs in the first vertex visited are assigned the first frequency of $F$, the second vertex visited determines the STRXs which get the second frequency assigned and so on.

In case a set $T$ requires more than one $\left(x_{T}>1\right)$ frequency, the vertex corresponding to $T$ has to be replaced by $x_{T}$ many copies. The new vertices are connected to each other with weight $a d(T, T)$ and to every other vertex $S$ with weight $a d(T, S)$. Consequently, every instance constructed in this manner has exactly $|F|+1$ vertices.

For an example, the example from Section "SFH" is continued. As stated in Section "Co-channel interference minimization", the optimal solution from the first stage was $x_{\left\{I_{1}, I_{2}\right\}}=x_{\left\{I_{3}\right\}}=2$ and $x_{T}=0$ for all other $T \subseteq \mathcal{S}$. Thus, $\bar{T}:=\left\{\left\{I_{1}, I_{2}\right\},\left\{I_{3}\right\}\right\}$. After calculating the induced adjacent channel interference $\operatorname{ad}\left(\left\{I_{1}, I_{2}\right\},\left\{I_{3}\right\}\right)$, the corresponding TSP instance is presented in Figure 2. There, each of the elements of $\bar{T}$ demands two frequencies such that the corresponding vertices have to be copied two times.

A result of this example could be as follows.

Tour:Dummy $\rightarrow\left\{I_{1}, I_{2}\right\} \rightarrow\left\{I_{3}\right\} \rightarrow\left\{I_{3}\right\} \rightarrow\left\{I_{1}, I_{2}\right\} \rightarrow$ Dummy

$\Rightarrow$ Total adjacent channel interference: $2 \cdot \operatorname{ad}\left(\left\{I_{1}, I_{2}\right\},\left\{I_{3}\right\}\right)$

$$
\begin{aligned}
\Rightarrow \text { Assignment: } & I_{1} \rightarrow\{1,4\} \\
I_{2} & \rightarrow\{1,4\} \\
I_{3} & \rightarrow\{2,3\}
\end{aligned}
$$$$
+\operatorname{ad}\left(\left\{I_{3}\right\},\left\{I_{3}\right\}\right)
$$

Depending on the values of $a d\left(I_{i}, I_{j}\right)$, this solution might be optimal.

Since the TSP is well studied [24], various mixed integer formulations are known, such as a sub-tour elimination formulation, which have proven to be applicable in practical circumstances. For a ready-to-use implementation, we refer to the SCIP implementation [25] or the CONCORDE solver [26]. Nevertheless, the formulation as derived above does not impose a metric on the resulting 


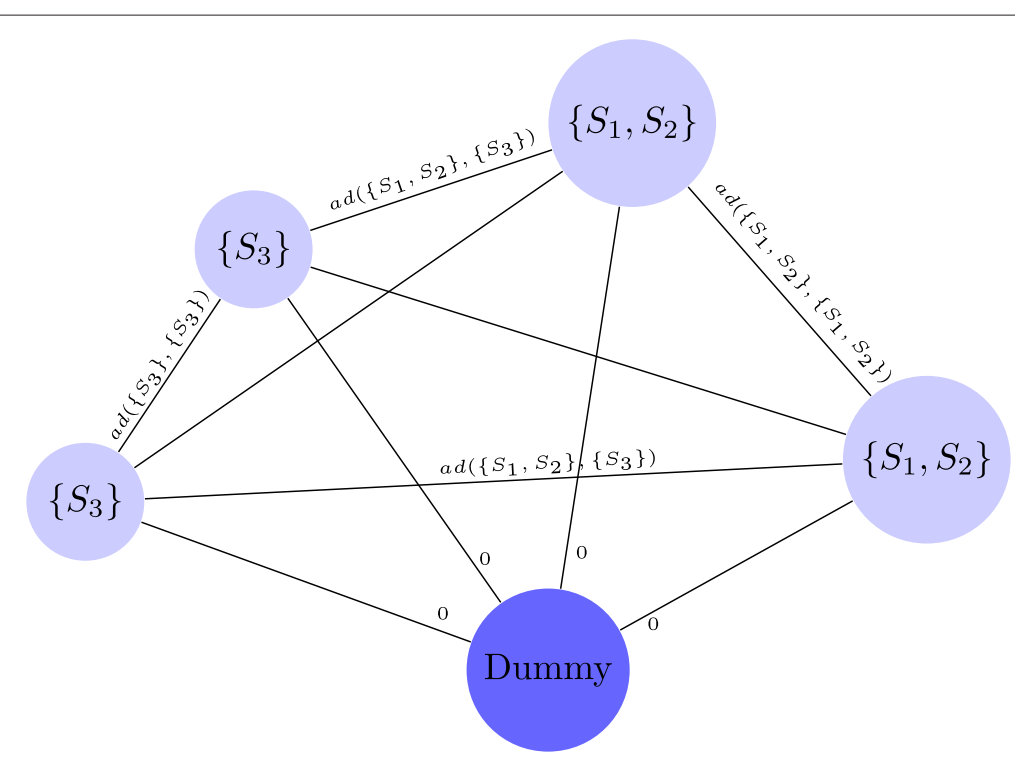

Figure 2 Second Stage Problem as TSP. Example of the TSP formulation of the second stage problem.

graph, such that these implementations cannot be used straight forward. As a result, we stick to the sub-tour elimination formulation in this matter. The sub-tour elimination formulation contains exponentially many constraints such that a separation approach is preferable. Separation of cutting planes means that the LP is only solved on a subset of all constraints. When a solution is available, it is checked whether an inequality is violated, which is in return added to the LP again and the process restarts. If no violated inequality exists, the LP-solution must be optimal. Similar as with the column generation approach, this concept helps to bypass memory limitations arising through exponentially many constraints. In fact, cutting planes and column generation are dual concepts. Again, the complexity of solving the LP relaxation depends on the computational complexity of the separating problem [21]. In this case, it can be solved, i.e., recognizing a violated sub-tour, in polynomial time. For more detailed information on cutting plane algorithms, we refer to [27].

In the context of MILP, cutting plane algorithms are mostly used to solve the linear relaxation of MILPs. For obtaining feasible integer solutions, it is necessary to combine cutting planes with a branching approach, i.e., every node is solved by cutting planes and it is then branched for integrality. Hereby, in any node different cutting planes might be added. On the whole, the combined method is called branch \& cut.

\section{Computational results}

In the following, it is explained why the decomposition into two stages provides more meaning-full insights than the first formulation. Nevertheless, an optimal solution cannot be guaranteed.

\section{Implementation details}

The first stage problem [FAPH1] is degenerated. To be more precise, the problem can be treated by primal heuristics rather easily, because of the similarity to the classic FAP, but an optimal solution by a branch and price algorithm cannot be guaranteed. This is explained in the following and the two main reasons are pointed out. In general, there are two main problems, the first corresponds to the incorporation of priced variables and the second corresponds to the solution of the pricing problem.

At first, we are describing the first reason. Within a branch and price algorithm, assume a variable given that is, in theory, improving the current basic solution (i.e., it has negative reduced costs). In general, these variables are expected to enter the next basic solution in the simplex algorithm. Nevertheless, in the [FAPH1] problem it may not be used in the next basic solution, i.e., its value remains at zero. This is based on the fact that the constraints are rather strict in the following sense: Assigning a value above zero to one variable may force other variables to change their values as well. These other variables are called correcting variables in the following, since they "correct" the changes the improving variable induces if used basic solution. In a column generation approach, these correcting variables may not be available yet such that the priced variable cannot be used. Additionally, these correcting variables may not have negative reduced costs in the current basic solution such that they are not recognized by the pricing problem. However, the reduced costs are updated after adding a new variable yielding that these variables will get negative reduced costs in the long run. Consequentially, solutions of the pricing problem 
are added successively until these correcting variables are all available. This may take arbitrarily many steps and is therefore not applicable for realistically sized instances. To our knowledge, no generic and efficient way of creating these correcting variables is known. In the following, we present some work around to this problem.

Given an improving variable, it is possible to create correcting variables heuristically. For this purpose, some modifications of construction heuristics, e.g., some DSATUR variation $[19,28]$ suffice to create enough surrounding variables such that the improving variable can potentially have a value above zero in the next basic solution. This means, given an improving variable, a totally new assignment based on this variable is created and the remaining variables are also added to the problem. Obviously, these surrounding variables may not form the best basic solution, with the priced variable contained, concerning the objective value. Further and especially concerning fractional solutions within the linear relaxation, the surrounding variables may not allow to use the improving variable to its full extend, compared to the best possible usage. Additional, since every assignment contains $|F|$ variables (compare constraint (6c)), adding one improving variable by adding $|F|-1$ others as well causes a certain inefficiency or redundancy. Consequentially, the solution process is slowed down. On the other hand, with these surrounding variables an improvement process can be constructed (see Section "Test results"), in which the improving variables are really used and at every pricing round, a primal solution is constructed. Hence, a pool of feasible integer solutions is generated which is a clear advantage over the [FAPSFH] formulation.

The second reason why no optimal solution can be claimed is the difficulty of the pricing problem. While it can be solved with reasonable effort at the beginning of the pricing process (e.g., by greedy heuristics) it gets increasingly hard to solve in the long run. Having variables in the order of $O(|\mathcal{S} \times \mathcal{S}|)$ and only searching for a minimum weighted (edge and node) subgraph makes up for a very difficult problem. As a result, the thorough solution of the pricing-problem can slow down the solution process significantly in the long run.

All in all, these are the two main reasons, why the solution process is slowed down, such that no optimal solution, not even of the linear relaxation can be determined for most test instances presented in this article.

This slow performance is often observed in column generation algorithms. Measure for obtaining speedups are known as stabilization techniques, some of which are presented in [29]. In general, these techniques allow an LP solution to violate the constraint matrix as long as a sufficient penalty is payed. At the beginning of column generation, this offers more (and potentially better) dual solutions, while in the optimum, the penalties for violating the constraints are too high, such that the optimum is the same as for the original problem. In our first stage problem, we assigned an additional "slack variable" (frequencies) to each constraint (6b), such that every STRX may use frequencies, not counting against the limit (6c) but with very high costs in the objective function. Consequentially, the pricing problem could be solved faster (at the beginning), such that there was no need for using heuristics only. On the other hand, the solutions started at a very high level of interference (compared to the original approach) and improved too slowly relative to the original approach, such that this stabilization techniques offered no significant gain.

Further stabilization techniques, e.g., presented in [30] advising alternative dual solutions for improving the pricing problem could not be used successfully either, since it is not trivial to find other dual solutions for our first stage problem. All in all, we are not aware of any stabilization techniques, significantly improving the first stage problem.

In addition to the problems presented above, no lower bounds or quality estimations on the linear relaxation can be provided. Like presented in [23], a lower bound $l b$ on the linear relaxation can be computed by means of the current LP solution $x^{*}$, the minimal reduced costs (the solution of the pricing problem) $r e d_{x^{*}}$ and an estimation on the number of used (i.e., non-zero) binary variables $|F|$ in each solution:

$$
l b=x^{*}-|F| \cdot \operatorname{red}_{x^{*}} .
$$

In a column generation framework, this lower bound converges (from below) to the optimum of the linear relaxation (when the solution is optimal, the reduced costs are equal to zero). Nevertheless, this bound needs some fading-in before it can produce non-trivial results. This means that $l b$ can be negative easily, while zero is a trivial lower bound in our problem. In our applications, no nontrivial results could be provided, for instances not being solved optimal. Hence, we are not able to give quality estimations on our primal solutions.

However, the second stage can be handled easier. The TSP instances in the second stage are very small with at most $|F|+1$ vertices. Hence, the TSP can be solved by the above formulation/separation approach. Contrary to the first stage, the second stage is solved optimally.

\section{Test environment}

The test instances have been taken from the COST256 project, see [18]. Since these scenarios are instances for the classic FAP, they do not incorporate SFH. Nevertheless, they offer the data on cell level, e.g., the TRXs are grouped into cells and the relations are given on this cell level and not for every single TRX. For creating SFHFAP instances, these cells have been interpreted as STRXs. 
Table 1 Overview scenario characteristics

\begin{tabular}{lllll}
\hline \multirow{2}{*}{ Name } & \multirow{2}{*}{ STRX } & \multicolumn{3}{c}{ Available Freq. } \\
\cline { 3 - 5 } & & $\mathbf{1 0 0 \%}$ & $\mathbf{5 0 \%}$ & $\mathbf{1 5 0 \%}$ \\
\hline Siemens1 & 506 & 75 & 37 & 112 \\
Bradford-0-Eplus & 1886 & 75 & 37 & 112 \\
Koeln & 264 & 50 & 25 & 75 \\
Swisscom & 148 & 68 & 34 & 102 \\
\hline
\end{tabular}

Overview on the used SFH-FAP instances. The second column describes the amount of STRXs, the third to fifth column denote the number of available frequencies.

The number of requested frequencies of each STRX $\left(k_{I}\right)$ has been set to the number of inbound TRXs plus four since research on the gain of SFH indicates that most improvement can already be accomplished by a fixed amount of additional (to the number of inbound TRXs per STRX) channels [6]. Furthermore, the interference relations have been adapted as described in (1) and (2). Separation and blocking constraints have been omitted as reasoned in the beginning of Section "Decomposition into two stages".

All computations were carried out on a $4 x \operatorname{Intel}(\mathrm{R})$ Xeon(R) CPU W3540@2.93 GHZ machine with 12 GB RAM and a SCIP 2.11 implementation (with CPLEX 12.4 as underlying solver). Especially, this means that columns are subject to aging, such that SCIP may delete unused columns after a certain time. This is important, since creating huge amounts of variables (because of the additional surrounding variables) would lead to a significant slowdown otherwise. However, the code has not been explicitly parallelized. As stop criterion a time limit of $100,000 \mathrm{~s}$ was set or until the available memory exhausted, depending on which condition occurred first.

\section{Test results}

Concerning the first stage, general statistics on the linear relaxation are given in Table 1 . The second column describes the amount of STRXs and the third to fifth column denote the number of available frequencies in three different cases. Further, Table 2 presents problem characteristics and solution values of its linear relaxation. The left numbers denote the amount of induced co-channel interference and the right numbers the amount of created variables $(k$ for 1,000). Additionally, Swisscom was solved optimal in all three versions while Bradford was aborted due to memory restrictions in the pricing problem. Having a closer look at statistics of the Siemens1 instance, the problems (degeneration) of the column generation approach become visible. As Figure 3 shows and like stated above, improving variables can be generated by solving [FAPHPR] during each pricing step. Adding these variables, together with heuristically generated correcting variables, can improve the objective function steadily (cf. Figure 4). Notably, both figures show a typical column generation behavior, i.e., a declining improvement. Nevertheless, the solution process is far too slow (cf. Table 2). While relatively many variables need to be added (the improving and the correcting variables amount to at last $|F|$ additional variables per pricing step), the improvement rate is diminishing. Consequentially not even the optimal LP solution is reachable in most cases.

Nevertheless, because of the heuristic construction of surrounding variables, a range of primal solutions is created. The best, which are later passed to the second stage are presented in Table 3 . There, the left numbers denote the amount of induced co-channel interference, the right numbers the time (in seconds) when the solution was found in the solution process.

Addressing the second stage, solution values are presented in Table 4. Given the best available, feasible solutions from the first stage, the best solutions of the corresponding second stage are reported. The left numbers denote the amount of induced adjacent channel interference, the right numbers the percentage of adjacent channel interference with respect to the total interference. As mentioned before, the second stage can be formulated as a TSP problem such that a generally good solvability, especially on small instances can be claimed. Hence no deepening research is invoked here.

Even though the methodological research is in focus here, some general conclusions from our results are drawn in the following. For the instances Bradford, Koeln and Swisscom the adjacent channel interference is marginal compared to co-channel interference ln the quality of the decomposition approach. Only for the Siemens1 instance

Table 2 Overview on first stage results

\begin{tabular}{|c|c|c|c|c|c|c|}
\hline \multirow[t]{2}{*}{ Name } & \multicolumn{3}{|c|}{ Best primal Sol. Val. } & \multicolumn{3}{|c|}{ \# Columns generated } \\
\hline & $100 \%$ & $50 \%$ & $150 \%$ & $100 \%$ & $50 \%$ & $150 \%$ \\
\hline Siemens1 & 1.18 & 22.81 & 0.02 & $85.0 k$ & $14.3 k$ & $216.0 k$ \\
\hline Bradford-0-Eplus & 133.94 & 468.08 & 55.03 & $5.0 k$ & $5.3 k$ & $5.8 k$ \\
\hline Koeln & 37.16 & 113.62 & 16.19 & $5.7 k$ & $1.6 k$ & $10.8 k$ \\
\hline Swisscom & 0.00 & 0.23 & 0.00 & $0.1 k$ & $0.0 k$ & $0.1 k$ \\
\hline
\end{tabular}

Results of the linear relaxation of the first stage after $100,000 \mathrm{~s}$ of computation time. The left numbers denote the amount of induced co-channel interference and the right the amount of created variables ( $k$ for 1,000$)$. 


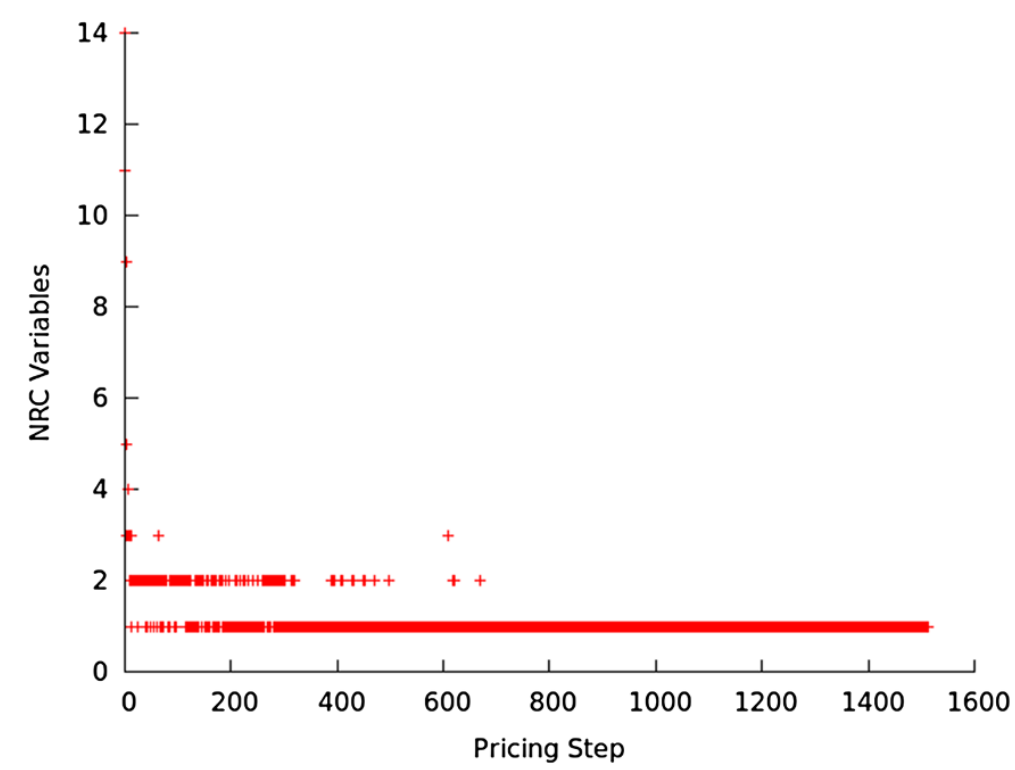

Figure 3 Improving variables found. The number of improving (negative reduced costs) variables per pricing step for the first stage problem in the Siemens 1 instance.

the adjacent channel interference is significant and an integrated approach might be beneficial.

Interference values are heavily dependent on the number of STRXs as well as of the number of available frequencies (combined with the particular interference relations). Obviously more STRXs lead to a higher amount of total interference while more frequencies reduce the interference. However, concerning computational effort it is to say that the amount of STRXs and the amount of frequencies determine the difficulty of the first stage whereas the second is only dependent on the number of available frequencies.

In the following, we will shortly comment on the results of our approach in relation to other methods. Since we cannot provide a proof of optimality in most cases, we refer to Table 5 for a comparison between our results and results from a (exemplary) straight forward heuristic (for the first stage). These solutions are obtained by a direct

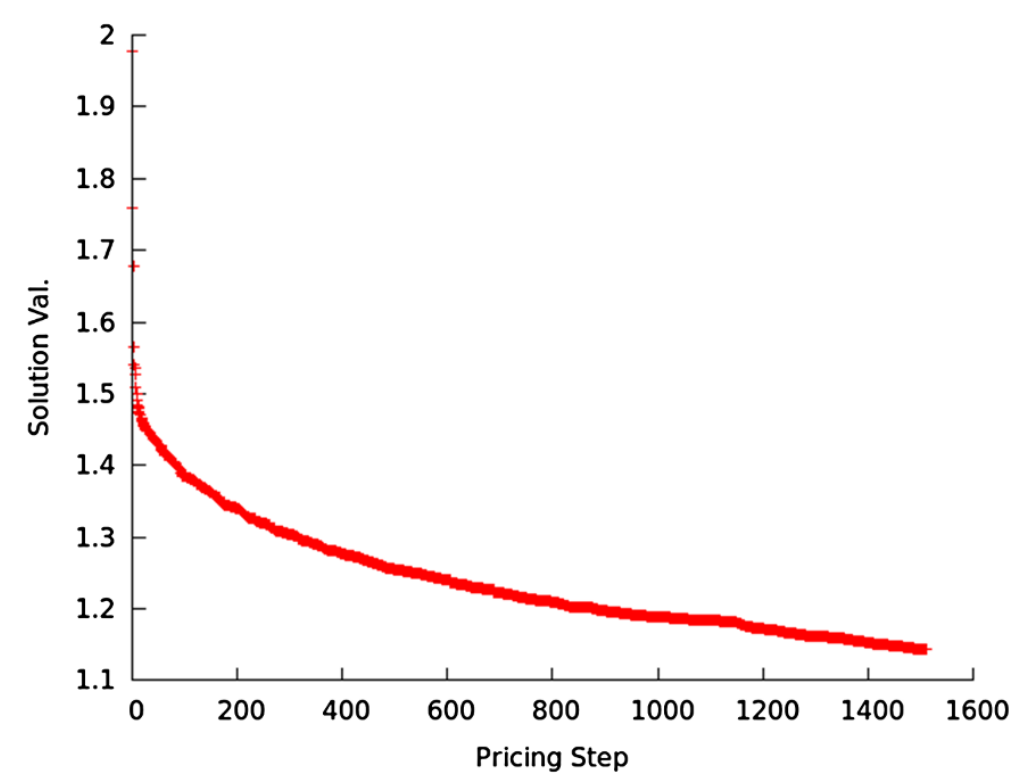

Figure 4 Solution Improvement per pricing step. The solution value relative to the pricing steps of the first stage problem for the Siemens 1 instance. 
Table 3 First stage best primal solutions

\begin{tabular}{|c|c|c|c|c|c|c|}
\hline \multirow[t]{2}{*}{ Name } & \multicolumn{3}{|c|}{ Best primal Sol. Val. } & \multicolumn{3}{|c|}{ Time (s) } \\
\hline & $100 \%$ & $50 \%$ & $150 \%$ & $100 \%$ & $50 \%$ & $150 \%$ \\
\hline Siemens 1 & 1.62 & 23.27 & 0.11 & $5.5 k$ & $0.2 k$ & $11.0 k$ \\
\hline Bradford-0-Eplus & 134.87 & 468.61 & 55.57 & $4.0 k$ & $2.2 k$ & $1.3 k$ \\
\hline Koeln & 39.32 & 115.14 & 17.98 & $0.0 k$ & $0.0 k$ & $1.0 k$ \\
\hline Swisscom & 0.00 & 0.68 & 0.00 & $0.0 k$ & $5.0 k$ & $0.0 k$ \\
\hline
\end{tabular}

The objective value of the best known integer solutions. The left numbers denote the amount of induced co-channel interference, the right numbers the time (in seconds) when the solution was found in the solution process.

greedy (DSATUR) heuristic for the first stage problem and then processed by the second stage. As one can see, the results obtained by the means of mixed integer programming are always better in the first stage. Because of the two staged approach, a better first stage solution does not correspond to a better second stage solution, such that the same cannot be claimed for the second stage. Nevertheless, only one instance was solved worse and the overall solution of both stages is always but one time better than the solutions produced by the greedy method in the first stage. However, this can obviously not be claimed for all heuristics, but this emphasizes the viability of mixed integer programming for such problems. Especially since each heuristic can be incorporated into a mixed integer programming approach, combining the strength of both approaches.

\section{Comparison to FAP}

At the beginning of this article, SFH was introduced and motivated on the background of the standard FAP. In this section, the above-presented results are related to the results of FAP, presented at [18]. Both problems are similar and produce interference results. However, these interference amounts are not straight forward comparable. Without going into technical details, SFH benefits from frequency- and interference-diversity and in the end of error recognition codes. Especially this means, that SFH networks can have single time-frames on single connections with very high interference without harming the overall speech quality, as long as the other time-frames offer a connection "good enough". The incorporation of random effects offers a gain towards the classic FAP beyond the pure measure of interference. However, this randomization formally leads to higher interference amounts of the assignment, regarding total numbers. This is explained in the following.

The FAP considers an optimal, overall assignment in every time-frame. In slow-frequency hopping, the assignment is changed at every time-frame, resulting in most time-frames not having optimal assignment. Every single time-frame is equal or worse compared to each FAP timeframe, concerning interference values but no frequency hopping gains. Consequently, the total expected interference in SFH-Networks must be higher compared to FAP networks. This can be illustrated by the following example: Consider the "tiny" instance from the FAP website, with the frequency spectrum set to $\{5, \ldots, 11\}$ and the $k_{I}$ values set to one (equal to FAP) and three, respectively. This leads to the following solutions:

Solution $k=1$ :

$$
\begin{aligned}
& x_{1,6,7}=x_{3,4}=x_{3,4,5}=x_{7}=1, \quad x_{2}=3 \\
& \text { Interference }=0 ;
\end{aligned}
$$

Solution $k=3$ :

$$
\begin{aligned}
& x_{2}=x_{2,3}=x_{3,4}=1, \quad x_{1,2,6,7}=x_{3,4,5}=2 \\
& \text { Interference }=2 \cdot \operatorname{Co}(\{1,2,6,7\})+1 \\
& \quad \times \operatorname{co}(\{2,7\})=2 \cdot 0.03+0.03=0.09
\end{aligned}
$$

\begin{tabular}{|c|c|c|c|c|c|c|}
\hline \multirow[t]{2}{*}{ Name } & \multicolumn{3}{|c|}{ Best primal Sol. Val. } & \multicolumn{3}{|c|}{$\%$ of total interference } \\
\hline & $100 \%$ & $50 \%$ & $150 \%$ & $100 \%$ & $50 \%$ & $150 \%$ \\
\hline Siemens 1 & 3.17 & 9.83 & 1.37 & 0.66 & 0.30 & 0.93 \\
\hline Bradford-0-Eplus & 8.34 & 20.78 & 4.33 & 0.06 & 0.04 & 0.07 \\
\hline Koeln & 1.11 & 3.46 & 0.42 & 0.03 & 0.03 & 0.02 \\
\hline Swisscom & 0.00 & 0.00 & 0.00 & - & 0.00 & - \\
\hline
\end{tabular}

Obviously the instance where $k=1$ has a lower frequency reuse factor, the average set size of STRXs sharing a frequency is smaller compared to the other case. Since the

Table 4 Overview second stage results

Given the best available, feasible solutions from the first stage (Table 3), the best solutions of the corresponding second stage. The left numbers denote the amount of induced adjacent channel interference, the right the percentage of adjacent channel interference of the total interference. 
Table 5 Direct heuristic results

\begin{tabular}{llllll}
\hline Name & Frequencies & First stage & Second stage & Total greedy & Total pricing \\
\hline Siemens1 & 75 & 1.98 & 3.39 & 5.37 & $\mathbf{4 . 7 9}$ \\
Siemens1 & 37 & 24.12 & 10.01 & 34.13 & $\mathbf{3 3 . 1 0}$ \\
Siemens1 & 112 & 0.18 & 1.24 & $\mathbf{1 . 4 2}$ & 1.48 \\
Bradford & 75 & 140.82 & 8.79 & 149.61 & $\mathbf{1 4 3 . 2 1}$ \\
Bradford & 37 & 470.51 & 20.32 & 490.83 & $\mathbf{4 8 9 . 3 9}$ \\
Bradford & 112 & 56.79 & 4.29 & 61.08 & $\mathbf{5 9 . 9 0}$ \\
Koeln & 50 & 40.36 & 1.13 & 41.49 & $\mathbf{4 0 . 4 3}$ \\
Koeln & 25 & 116.23 & 3.36 & 119.69 & $\mathbf{1 1 8 . 6 0}$ \\
Koeln & 75 & 19.18 & 0.42 & 19.60 & $\mathbf{0 . 0 0}$ \\
Swisscom & 68 & 0.00 & 0.00 & 1.04 & $\mathbf{0 . 0 0}$ \\
Swisscom & 34 & 1.04 & 0.00 & 0.00 & $\mathbf{0 . 6 8}$ \\
Swisscom & 102 & 0.00 & 0.00 & $\mathbf{0 . 0 0}$ \\
\hline
\end{tabular}

Comparison to results obtained by a straight forward greedy-heuristic for the first stage. The overall best results per instance have been marked bold.

chance of interference rises quadratically in the amount of STRX in a single set, the overall interference level rises (though every single potential interference value is strictly lower) if $k$ increases. In other words, with a relatively low $k$ value it is possible to form sets of STRX having very few (if any) interference relations not equal to zero while at higher $k$ values, i.e., demanding more frequencies to hop, increases the set size and therefore the chance of strictly positive interference values dramatically. In the above context, this means that inducing randomization will increase the probability of having a suboptimal assignment in one time-frame, thus increasing the expected interference.

In consequence, more (positive) interference values are taken into account in SFH compared to FAP. Although FAPH has smaller interference values per "link", see (1) and (2), this leads to an overall higher number of expected interference, therefore making a comparison by pure interference values misleading.

\section{Conclusions}

Drawing a final conclusion on the presented research, the concept of SFH-FAP can be modeled by means of mixed integer programming, similar to the classical FAP. Nevertheless, realistically sized instances cannot be solved and demand for more sophisticated approaches. Here, a decomposition into two stages was proposed such that the huge size of the problems can be engaged by a column generation and separating approach, respectively. While this decomposition does not allow to obtain optimal results in the first stage, by a sensible implementation, a range of primal solutions/assignments could be produced which can be passed on to the second stage so that the assignment can be finalized there. However, the problem is extremely hard so solve, as well in the perspective of complexity theory as by practical aspects. Exponentially many variables lead to column generation, whereas the pricing problem is, because if its generality, very hard to solve. The solutions of the pricing problem are (even with stabilization techniques) not trivially incorporated into the first stage problem, such that the improvement rate is very slow and the optimum is not reachable.

Since the obtained results are not optimal, the whole effort has a heuristic character, only. Hence, this approach might be, because of the rather high computational effort, inferior to a straight forward heuristic solution without invoking the means of mixed integer programming. Although we cannot claim an optimal solution, the bottleneck of this approach could be pointed out, namely a (fast) solution of the pricing problems as well as the incorporation of newly priced variables into new basic solutions. Future research, which bypasses this problem, could lead to an optimal solution of the first stage such that the complete frequency assignment in SFH networks could be done evidently close to optimal.

\section{Competing interests}

Both authors declare that they have no competing interests.

Received: 15 February 2012 Accepted: 25 July 2012

Published: 14 August 2012

\section{References}

1. ST Chung, A Goldsmith, Degrees of freedom in adaptive modulation: a unified view. IEEE VTS 53rd Veh. Technol. Conf. 2, 1267-1271 (2001)

2. X Qiu, K Chawla, On the performance of adaptive modulation in cellular systems. IEEE Trans. Commun. 47, 884-895 (1999)

3. L Toni, A Conti, Does Fast Adaptive Modulation Always Outperform Slow Adaptive Modulation? IEEE Trans. Wirel. Commun. 10, 1504-1513 (2011)

4. H Bogucka, A Conti, Degrees of freedom for energy savings in practical adaptive wireless systems. IEEE Commun. Mag. 49, 38-45 (2011)

5. M Chiani, A Conti, R Verdone, Partial compensation signal-level-based up-link power control to extend terminal battery duration. IEEE Trans. Veh. Technol. 50, 1125-1131 (2001) 
6. H Olofsson, J Naeslund, J Skold, Interference diversity gain in frequency hopping GSM. 45th Veh. Technol. Conf. 1, 102-106 (1995)

7. M Chiani, E Agrati, $O$ Andrisano, An analytical approach to evaluate service coverage in slow frequency-hopping mobile radio systems. IEEE Trans. Commun. 49(8), 1447-1456 (2001)

8. M Chiani, A Conti, O Andrisano, Outage evaluation for slow frequency-hopping mobile radio systems. IEEE Trans. Commun. 47(12), 1865-1874 (1999)

9. KI Aardal, CPM van Hoesel, AMCA Koster, C Mannino, A Sassano, Models and solution techniques for the frequency assignment problem. Ann. Oper. Res. 153, 79-129 (2007). doi:10.1007/s10479-007-0178-0

10. B Jaumard, O Marcotte, C Meyer, T Vovor, Comparison of column generation models for channel assignment in cellular networks. Discrete Appl. Math. 112(1-3), 217-240 (2001)

11. B Jaumard, O Marcotte, C Meyer, T Vovor, Erratum to "Comparison of column generation models for channel assignment in cellular networks". Discrete Appl. Math. 118(3), 299-322 (2002)

12. TD Hemazro, B Jaumard, O Marcotte, A column generation and branch-and-cut algorithm for the channel assignment problem. Comput. Oper. Res. 35(4), 1204-1226 (2008)

13. A Mehrotra, M Trick, A column generation approach for graph coloring. INFORMS J. Comput. 8(4), 344-354 (1995)

14. J Moon, L Hughes, D Smith, Assignment of frequency lists in frequency hopping networks. IEEE Trans. Veh. Technol. 54(3), 1147-1159 (2005)

15. T Nielsen, J Wigard, PH Michaelsen, P Mogensen, Resource allocation in a frequency hopping PCS1900/GSM/DCS1800 type of network. 49th IEEE Veh. Technol. Conf. 1, 209-214 (1999)

16. P Björklund, P Värbrand, D Yuan, Optimal frequency planning in mobile networks with frequency hopping. Comput. Oper. Res. 32, 169-186 (2005)

17. M Tieves, Frequency assignments in slow frequency-hopping gsm networks-a MIP approach. Master's thesis, RWTH Aachen, (2011). http:// www.math2.rwth-aachen.de/de/forschung/publ

18. A Koster, A Eisenblätter. http://fap.zib.de, 2001-2012

19. A Eisenblätter, Frequency assignment in GSM networks: models, heuristics, and lower bounds. PhD thesis, TU Berlin, 2001

20. A Mehrotra, M Trick, in Extending the Horizons: Advances in Computing, Optimization, and Decision Technologies A branch-and-price approach for graph multi-coloring. (Springer, New York, 2007), pp. 15-29

21. M Grötschel, L Lovász, A Schrijver, Geometric Algorithms and Combinatorial Optimization, Volume 2 of Algorithms and Combinatorics (Springer, New York, 1988)

22. D Johnson, M Garey, Computers and Intractability: A Guide to the Theory of NP-Completeness (W. H. Freeman, San Francisco, 1979)

23. G Desaulniers, J Desrosiers, M Solomon, Column Generation (Springer, New York, 2005)

24. D Applegate, R Bixby, V Chvatal, W Cook, The Traveling Salesman Problem (Princeton University Press, Princeton, NJ , 2006)

25. T Achterberg, T Berthold, Scip tsp example. http://scip.zib.de/doc/ examples/TSP/index.html

26. Concorde Solver. http://www.tsp.gatech.edu/index.html

27. L Wolsey, Integer Programming (John Wiley and Sons, New York, 1998)

28. D Brélaz, New methods to color the vertices of a graph. Commun. ACM. 22, 251-256 (1979)

29. O Du Merle, D Villeneuve, J Desrosiers, P Hansen, Stabilized column generation. Discrete Math. 194, 229-237 (1999)

30. M Leitner, M Ruthmair, G Raidl, On stabilizing branch-and-price for constrained tree problems. Technical Report, Institute of Computer Graphics nad Algorithms, Vienna, 2011

Cite this article as: Koster and Tieves: Column generation for frequency assignment in slow frequency hopping networks. EURASIP Journal on Wireless Communications and Networking 2012 2012:253. 